\title{
1 Olive oil phenolic compounds and high-density lipoprotein function
}

2 Hernáez $\mathrm{A}^{1,2,3}$, Farràs $\mathrm{M}^{1,2}$, Fitó $\mathrm{M}^{1,2, *}$

3

$4{ }^{1}$ Cardiovascular Risk and Nutrition Research Group, REGICOR Study Group, Hospital

5 del Mar Medical Research Institute (IMIM), Barcelona, Spain

$6 \quad{ }^{2}$ CIBER de Fisiopatología de la Nutrición y la Obesidad (CIBEROBN), Instituto de 7 Salud Carlos III, Madrid, Spain

$8{ }^{3}$ Ph.D Program of Food Science and Nutrition, Universitat de Barcelona, Barcelona, 9 Spain

11 Corresponding author

12 Montserrat Fitó, MD, PhD

13 Cardiovascular Risk and Nutrition Research Group

14 CIBER de Fisiopatología de la Nutrición y la Obesidad (CIBEROBN)

15 Hospital del Mar Medical Research Institute (IMIM)

16 Carrer Doctor Aiguader, 88, 08003, Barcelona, Spain

17 Telephone number: (+34) 933160724

18 Fax: (+34) 933160796

19 E-mail: $\underline{\text { mfito@imim.es }}$ 
ABSTRACT

\section{Purpose of review}

The functional capacities of high-density lipoproteins (HDLs) reflect the physiological role of the particle better than the quantity of HDL cholesterol. Due to its phenolic compounds, the consumption of virgin olive oil has emerged as a promising therapy to promote these capacities. This review highlights the human studies that explain these benefits and explores some possible mechanisms.

\section{Recent findings}

The consumption of olive oil phenolic compounds increased the ability of HDLs to pick up cholesterol excess in peripheral cells (the cholesterol efflux capacity). Olive oil phenolic compounds have also been shown to improve HDL antioxidant capacities and some anti-inflammatory traits. These changes respond to an improvement of HDL oxidative status and composition.

Summary

Novel strategies to increase HDL functional capacities are in demand from clinicians.

The attainment of a fully-functional HDL through dietary or lifestyle changes is a priority in cardiovascular research. Within this context, the consumption of virgin olive oil, due to its phenolic compounds, may be a relevant protective approach. Further studies in large-scale, randomized controlled trials are, however, required to confirm these effects in HDL functionality.

\section{KEYWORDS}

43 HDL function, olive oil phenolic compounds, cholesterol efflux capacity, HDL antioxidant capacity 
45 ABBREVIATIONS

46 ApoA-I: apolipoprotein A-I

47 CEC: cholesterol efflux capacity

48 CVD: cardiovascular disease

49 FVOO: functional virgin olive oil, enriched with olive oil phenolic compounds

50 FVOOT: functional virgin olive oil, enriched with olive oil phenolic compounds +

51 phenols from thyme

52 HDL: high-density lipoprotein

53 HDL-C: high-density lipoprotein cholesterol

54 LCAT: lecithin-cholesterol acyltransferase

55 LDL: low-density lipoprotein

56 MD: Mediterranean Diet

57 PAF-AH: platelet-activating factor acetylhydrolase

58 PON-1: paraoxoase-1

59 PPAR: peroxisome proliferator-activated receptor

60 VOO: virgin olive oil 
62 High-density lipoprotein (HDL) cholesterol (HDL-C) levels are inversely and independently associated with cardiovascular disease (CVD) [1]. Several interventions, based on pharmacological and natural products, have been able to increase HDL-C concentrations although high levels have not always been associated with low cardiovascular risk [2]. Moreover, pharmacologically raised HDL-C levels have not always led to a decrease in cardiovascular risk and some studies have even reported an increased mortality risk [3]. The physiological role of HDL seems, therefore, to be better reflected by its function than HDL-C quantity. HDLs play a central role in reverse cholesterol transport. They remove excess cholesterol from peripheral cells (the cholesterol efflux capacity, CEC) and transport it to the liver for further metabolism and excretion [4]. CEC has been shown to predict coronary event incidence [5] and be inversely related to the development of early atherosclerosis [6]. HDLs present other atheroprotective capacities: they counteract the major role in its protective effects on CVD [8]. In observational studies adherence to the MD decreased the development of chronic CVD [9] whilst intervention studies demonstrated the beneficial effect of this diet on CVD primary and secondary prevention $[10,11]$. Our group reported that the consumption of virgin olive oil (VOO) increased HDL-C and decreased in vivo lipid oxidative damage. This effect was dosedependently associated with the olive oil phenolic compound content [12]. Taking into account the rise in HDL-C quantity, an increase in the functional capacities of HDL due to the consumption of VOO could be expected. The whole matrix of phenolics in olive 
oil is complex and diverse (Table 1) [13] and would be responsible for these potentially beneficial effects.

Our aim is to review the reported benefits of the consumption of phenolic compounds in VOO on the functionality of HDLs in humans. Further discussion of the possible related mechanisms is also provided.

\section{HDL CHOLESTEROL EFFLUX CAPACITY}

The consumption of phenolic compounds has increased CEC in some in vivo studies [14]. With respect to olive oil phenolic compounds, our group provided first-level evidence for the first time in humans of an increase in CEC after the consumption of a real-life dose of VOO $(366 \mathrm{mg} / \mathrm{kg})$ [15**]. To explain this change, we observed, on the one hand, an increase in the biological metabolites of olive oil phenolic compounds bound to the HDLs (hydroxytyrosol sulfate, and homovanillic acid sulfate and glucuronate). These compounds could exert a local antioxidant protection in HDLs $\left[16,17,18^{*}\right]$ that may prevent oxidative modifications of the apolipoprotein A-I (ApoAI), the main HDL protein involved in CEC [4], and of other HDL proteins. Such protection would also avoid oxidative modifications of HDL lipids, making the lipoprotein more fluid and thus more functional [19]. Improvements in CEC and HDL fluidity have also been described in a non-controlled trial with VOO in healthy volunteers [20]. On the other hand, we found a decrease in the relative content of triglycerides in the HDL core. A triglyceride-poor HDL core is related to a more stable conformation of ApoA-I in the lipoprotein [21] which could lead to better HDL capacity to perform CEC.

Functional VOOs are also a promising therapy to improve CEC. Our group recently developed a randomized controlled trial in hypercholesterolemic individuals to test the 
111 effects on the HDL characteristics related to CEC of two functional VOOs: one

112 enriched with olive oil phenolic compounds (500 mg/kg; FVOO) and another with

113 phenolic compounds from olive $(250 \mathrm{mg} / \mathrm{kg})$ plus additional complementary phenolic

114 compounds from thyme (250 mg/kg) (total: $500 \mathrm{mg} / \mathrm{kg}$; FVOOT), compared with a

115 standard VOO $(80 \mathrm{mg} / \mathrm{kg})[22 *]$. The functional oils increased the quantity and

116 bioavailability of the phenolic compounds in the real food matrix without raising the

117 final fat intake [23]. Three main effects were observed. First, an increase in the lecithin-

118 cholesterol acyltransferase (LCAT) mass in plasma after the FVOOT intervention

versus the control VOO was reported. LCAT mediates the esterification of free

cholesterol and its migration into the particle core. This process leads to the conversion of the immature HDLs into small particles and, finally, into larger ones [4]. The increase in LCAT mass could, therefore, be linked to a decrease in the relative content of free cholesterol and an increase in the relative content of phospholipids in the HDL surface, both observed after the FVOOT intervention versus the control VOO [22*]. Such changes may result in more fluid HDLs, indirectly associated with a greater CEC [19]. Second, in a pooling sample proteomic approach, we observed an increase in the ApoA-I content in HDL after the consumption of VOO and both functional VOOs [24*]. ApoA-I-rich HDLs may be more efficient in exerting CEC since ApoA-I is the main HDL protein involved in the process. Finally, in the same proteomic approach, we also found an increase in the content of affamin in HDL after the FVOOT intervention [24*]. Affamin is a transporter of $\alpha$-tocopherol and, as a result, we would expect an increased $\alpha$-tocopherol content in HDL which could complement the local antioxidant protection of the phenolic compounds.

CEC can also be modulated by the direct effect of olive oil phenolic compounds on CEC-related gene expression. In a postprandial study with pre-/hypertensive 
individuals, a real-life dose of phenolic compound-enriched olive oil (961 mg/kg)

enhanced the gene expression of transmembrane cholesterol transporters (ABCA1, SR-

B1), and some transcription factors related to the peroxisome proliferator-activated (289 $\mathrm{mg} / \mathrm{kg}$ ) [25]. The improvement in ABCA1 and SR-B1 expression could be mediated through the up-regulation of the PPAR-dependent pathway [26]. We additionally reported an augmentation in the PPAR-related gene expression in a previous postprandial study with an acute dose of $50 \mathrm{~mL}$ of VOO [27]. With respect to long-term studies, similar increases in the expression of some transmembrane healthy individuals [28].

\section{HDL ANTIOXIDANT ACTIVITY}

HDLs protect LDLs against oxidative modifications; a relevant property since oxidized LDLs are a key trigger for the onset of atherosclerotic plaque [29]. The consumption of VOO has been dose-dependently associated in vivo with a decrease in oxidized LDLs and the platelet-activating factor acetylhydrolase (PAF-AH), and the indirect protection 
161 As previously commented, the consumption of olive oil phenolic compounds may enhance the ApoA-I functionality in HDL particles, preventing oxidative modifications of the protein and leading to a more stable conformation of ApoA-I in HDLs [15**]. These characteristic might also improve HDL capacity to pick up oxidized lipids [4]. In parallel, olive oil phenolic compounds could enhance the function of some HDLrelated antioxidant enzymes, such as PON1. PON1 is present in the circulation mainly linked to HDLs and is one of the principal agents involved in the hydrolysis of oxidized lipids in plasma [30]. PON1 antioxidant activity was enhanced in healthy humans after a 3-week VOO-rich intervention [17] and also significantly upgraded after the consumption of the previously described FVOOT [22*]. These improvements in PON1 function are in line with others found after supplementation with VOO in several murine models [31-33]. Three possible mechanisms may explain this increment in PON1 function. First, olive oil phenolics could increase the biosynthesis of PON1, since the consumption of VOO induced a rise in the PON1 plasma concentrations of healthy subjects $\left[18^{*}\right]$. The capacity of these phenolic compounds to stimulate the Nrf2dependent gene expression could indirectly enhance PON1 production [33]. Second, HDLs may become rich in PON1, in a similar way to their becoming abundant in PON3 (a PON1 genotypic isoform), as observed after the consumption of VOO and the previously described FVOOT [24*]. Finally, the consumption of VOO increased the HDL content of olive oil phenolic compound metabolites which may protect PON1 commented on an increment of LCAT mass after the consumption of the FVOOT when compared to a control VOO [22*]. In the case of the PAF-AH, no significant changes in PAF-AH quantity or activity in HDLs due to VOO have as yet been reported. 


\section{HDL ANTI-INFLAMMATORY AND VASOPROTECTIVE EFFECTS}

In addition to their CEC and antioxidant properties, HDLs are also considered to be relevant anti-inflammatory and vasoprotective agents. Endothelial dysfunction and the inflammatory responses of macrophages and endothelial cells, key factors for the perpetuation of atherosclerosis [29], all seem to be counteracted by HDLs [7]. VOO consumption has been shown to be highly protective for vascular response and endothelial integrity, as observed in a number of VOO-rich interventions in humans [34-36]. HDLs could act as transporters of several derivatives of olive oil phenolic compounds to the endothelial cells where they may prevent oxidative damage in cell mitochondria and preserve the production of nitric oxide, as described in vitro [35,37]. Regarding HDL anti-inflammatory capacity, the consumption of VOO increased the ability of HDLs to block the secretion of intracellular adhesion molecule-1 and the adhesion of monocytes to endothelial cells in healthy humans [18*]. Such an enhancement could be due to two hypothetical mechanisms. First, the improvement in HDL antioxidant function caused by olive oil phenolic compounds may partially explain these benefits. HDL antioxidant and anti-inflammatory functions are intimately related [7] and PON1 is one of the principal agents involved [7,30]. In agreement with this, the anti-inflammatory properties of PON1 activity increased significantly after the consumption of VOO in healthy subjects [18*]. Second, the consumption of VOO may enhance HDL functionality by decreasing the levels of acute-phase proteins in the lipoprotein. Inflammatory states increase the content of acute-phase proteins in HDLs, transforming the lipoproteins into pro-inflammatory, dysfunctional particles [4]. The consumption of different VOOs [24*] and a VOO-rich MD [38] has been reported to 
decrease the content of acute-phase proteins in HDLs and may, therefore, promote a less pro-inflammatory state of the particles.

212

\section{OTHER HDL-RELATED PROPERTIES}

214 In addition to the functional properties of HDLs there are other novel characteristics

215 related to their quantity and quality. The number of HDL particles in circulation, determined by NMR-spectroscopy, has shown to be a promising biomarker of HDL concentration, for instance in the prediction of CVD incidence [39]. A short-term consumption of VOO in healthy individuals induced a non-significant increasing trend in HDL particle number [15**]. This protective effect was significantly confirmed after a long-term consumption of an MD supplemented with nuts [40]. A rise in systemic ApoA-I levels, such as those reported after some VOO-based dietary interventions in humans [41,42], may justify these changes. Mechanistically, a regular intake of VOO may enhance the intestinal expression of Apoal gene, as observed in a rat model [43]. Olive oil phenolic compounds may also decrease ApoA-I degradation, as described in individuals whose HDL-C concentrations increased after a short-term consumption of an MD [44].

High CVD risk patients usually present a profile characterized by low levels of large HDLs, high levels of small HDLs, and variable values of lipid-poor/lipid-free HDLs (the pre- $\beta$ fraction, the most effective one for CEC) [45]. The consumption of VOO has shown to increase large HDLs and decrease small ones [15**]. The change towards greater HDL size has been confirmed after the consumption of an FVOOT [22*], a VOO-rich MD [40,46], and in a rat model after supplementation with VOO [47]. There is however some controversy with respect to this issue. Some authors consider that the pre- $\beta$ HDL and lipid-free ApoA-I are the more functional particles [48]. In contrast, a 
number of in vitro studies indicate that small HDLs have similar effects to the large ones [49]. Furthermore, increased levels of small HDLs in plasma may indicate an aberration in HDL maturation and decreased reverse cholesterol transport [4]. Large HDLs also bind better to the ABCG1 and SR-B1 cholesterol transporters, promoting cholesterol efflux via these receptors [4]. Moreover, some HDL physicochemical modifications (such as the ones in inflammatory states) can transform the lipoprotein into a small, dysfunctional particle [4]. As a result, the interpretation of HDL size without taking into account the overall biochemical context is controversial. Finally, some VOO-based interventions, particularly the FVOOT, have increased the content of other active proteins in HDLs, such as that of apolipoprotein A-IV [24*]. This effect has also been observed in apoE-deficient mice after the consumption of a VOO-rich diet [31]. A rise in HDL apolipoprotein A-IV content may be cardioprotective since a decreased apolipoprotein A-IV content in HDLs appears in patients with stable or acute coronary syndrome [50].

\section{CONCLUSIONS}

HDL functions reflect the physiological role of the lipoprotein better than HDL-C quantity. As indicated in Figure 1, the intake of olive oil phenolic compounds resulted in an improvement in CEC, HDL antioxidant defenses, HDL size distribution, and other characteristics related to HDL quality. Olive oil phenolic compounds bound to HDLs, or surrounding the lipoprotein, improve their oxidative/inflammatory status which may justify an increase in HDL functionality. Modifications in HDL composition due to the consumption of VOO might also explain these changes. However, large-scale, randomized controlled trials with VOO-rich dietary interventions are required to 
259 definitively confirm the protective role of olive oil phenolic compounds in HDL

260 biological functions. 


\section{Key points}

- HDL function reflects the physiological role of the lipoprotein better than HDL cholesterol

- Lifestyle changes that may increase HDL functional capacities in vivo are in high demand from clinicians

- The phenolic compounds in virgin olive oil are able to improve HDL cholesterol efflux capacity

- Olive oil phenolic compounds also increase HDL antioxidant capacities

- Large-scale, long-term randomized controlled trials with virgin olive-rich dietary interventions are required to confirm the protective effects of olive oil phenolic compounds on HDL function

\section{Acknowledgements}

We would like to thank Stephanie Lonsdale for her assistance in editing the English text. CIBER de Fisiopatología de la Obesidad y Nutrición (CIBEROBN) is an initiative of the Instituto de Salud Carlos III, Madrid, Spain.

\section{Financial support and sponsorship}

A.H. received a fellowship from the Spanish Ministry of Education, Culture and Sport (FPU12/01318), M.Farràs was funded by an FPI fellowship (BES-2010-040766), and M.Fitó was funded by the Catalan Government and the Instituto de Salud Carlos IIIFEDER (FIS CP06/00100). This work was supported by the Instituto de Salud Carlos III-FEDER (CB06/03/0028, and FIS programs PI070759 and PI11/01647; Madrid, Spain), the Spanish Ministry of Economy and Competitiveness (AGL2009-13517-C03- 
28501 and AGL2012-40144-C03-01; Madrid, Spain) and AGAUR (2014-SGR-240;

286 Barcelona, Spain).

287

288 Conflicts of interest

289 None. 


\section{REFERENCES}

1. Castelli WP, Doyle JT, Gordon T, et al. HDL cholesterol and other lipids in coronary heart disease. The cooperative lipoprotein phenotyping study. Circulation 1977; 55:767-772.

2. Voight BF, Peloso GM, Orho-Melander M, et al. Plasma HDL cholesterol and risk of myocardial infarction: a mendelian randomisation study. Lancet 2012; 380:572-580.

3. Barter PJ, Caulfield M, Eriksson M, et al. Effects of torcetrapib in patients at high risk for coronary events. N Engl J Med 2007; 357:2109-2122.

4. Kontush A, Chapman MJ. Functionally defective high-density lipoprotein: a new therapeutic target at the crossroads of dyslipidemia, inflammation, and atherosclerosis. Pharmacol Rev 2006; 58:342-374.

5. Rohatgi A, Khera A, Berry JD, et al. HDL cholesterol efflux capacity and incident cardiovascular events. N Engl J Med 2014; 371:2383-2393.

6. Khera AV, Cuchel M, de la Llera-Moya M, et al. Cholesterol efflux capacity, high-density lipoprotein function, and atherosclerosis. N Engl J Med 2011, 364:127-135.

7. Besler C, Lüscher TF, Landmesser U. Molecular mechanisms of vascular effects of High-density lipoprotein: alterations in cardiovascular disease. EMBO Mol Med 2012; 4:251-268.

8. Guasch-Ferré M, Hu FB, Martínez-González MA, et al. Olive oil intake and risk of cardiovascular disease and mortality in the PREDIMED Study. BMC Med 2014, 12:78.

9. Trichopoulou A, Costacou T, Bamia C, Trichopoulos D. Adherence to a Mediterranean diet and survival in a Greek population. N Engl J Med 2003; 348:2599-2608.

10. Estruch R, Ros E, Salas-Salvadó J, et al. Primary prevention of cardiovascular disease with a Mediterranean diet. N Engl J Med 2013; 368:1279-1290.

11. De Lorgeril M, Salen P, Martin JL, et al. Mediterranean diet, traditional risk factors, and the rate of cardiovascular complications after myocardial infarction: final report of the Lyon Diet Heart Study. Circulation 1999; 99:779-785. 
12. Covas MI, Nyyssönen K, Poulsen HE, et al. The effect of polyphenols in olive oil on heart disease risk factors: a randomized trial. Ann Intern Med 2006; 145:333-341.

13. Lou-Bonafonte JM, Fitó M, Covas M-I, et al. HDL-related mechanisms of olive oil protection in cardiovascular disease. Curr Vasc Pharmacol 2012; 10:392-409.

14. Zhu Y, Huang X, Zhang Y, et al. Anthocyanin supplementation improves HDLassociated paraoxonase 1 activity and enhances cholesterol efflux capacity in subjects with hypercholesterolemia. J Clin Endocrinol Metab 2014; 99:561-569.

**15. Hernáez Á, Fernández-Castillejo S, Farràs M, et al. Olive oil polyphenols enhance high-density lipoprotein function in humans: a randomized controlled trial. Arterioscler Thromb Vasc Biol 2014; 34:2115-2119.

This is the first randomized controlled trial that shows an improvement in cholesterol efflux capacity, HDL oxidative status and HDL size distribution after the consumption of olive oil phenolic compounds.

16. Atzeri A, Lucas R, Incani A, et al. Hydroxytyrosol and tyrosol sulfate metabolites protect against the oxidized cholesterol pro-oxidant effect in Caco-2 human enterocyte-like cells. Food Funct 2015; doi:10.1039/c5fo00074b.

17. Cherki M, Derouiche A, Drissi A, et al. Consumption of argan oil may have an antiatherogenic effect by improving paraoxonase activities and antioxidant status: Intervention study in healthy men. Nutr Metab Cardiovasc Dis 2005; 15:352360.

*18. Loued S, Berrougui H, Componova P, et al. Extra-virgin olive oil consumption reduces the age-related decrease in HDL and paraoxonase 1 anti-inflammatory activities. Br J Nutr 2013; 110:1272-1284.

This is the first trial that shows an increase in PON1 levels and in HDL antiinflammatory properties after the consumption of a virgin olive oil.

19. Bonnefont-Rousselot D, Motta C, Khalil AO, et al. Physicochemical changes in human high-density lipoproteins (HDL) oxidized by gamma radiolysis-generated oxyradicals. Effect on their cholesterol effluxing capacity. Biochim Biophys Acta $1995 ; 1255: 23-30$.

20. Helal O, Berrougui H, Loued S, Khalil A. Extra-virgin olive oil consumption improves the capacity of HDL to mediate cholesterol efflux and increases ABCA1 and ABCG1 expression in human macrophages. Br J Nutr 2013; 109:1844-1855. 
21. Sparks DL, Davidson WS, Lund-Katz S, Phillips MC. Effects of the neutral lipid content of high density lipoprotein on apolipoprotein A-I structure and particle stability. J Biol Chem 1995; 270:26910-26917.

*22. Farràs M, Castañer O, Martín-Peláez S, et al. Complementary phenol-enriched olive oil improves HDL characteristics in hypercholesterolemic subjects. A randomised, double-blind, crossover, controlled trial. The VOHF study. Mol Nutr Food Res 2015 [In press].

This randomized controlled trial shows that functional olive oils, enriched in olive oil or thyme phenolic compounds, improve several characteristics related to HDL quality.

23. Rubió L, Motilva MJ, Macià A, et al. Development of a phenol-enriched olive oil with both its own phenolic compounds and complementary phenols from thyme. J Agric Food Chem 2012; 60:3105-3112.

*24. Pedret A, Catalán Ú, Fernández-Castillejo S, et al. Impact of Virgin Olive Oil and Phenol-Enriched Virgin Olive Oils on the HDL Proteome in Hypercholesterolemic Subjects: A Double Blind, Randomized, Controlled, Cross-Over Clinical Trial (VOHF Study). PLoS One 2015; 10:e0129160. This study shows that functional olive oils, enriched in olive oil or thyme phenolic compounds, modify the proteomic composition of HDL particles.

25. Farràs M, Valls RM, Fernández-Castillejo S, et al. Olive oil polyphenols enhance the expression of cholesterol efflux related genes in vivo in humans. A randomized controlled trial. J Nutr Biochem 2013; 24:1334-1339.

26. Hamblin M, Chang L, Fan Y et al. PPARs and the cardiovascular system. Antioxid Redox Signal 2009; 11:1415-1452.

27. Konstantinidou V, Khymenets O, Covas MI, et al. Time course of changes in the expression of insulin sensitivity-related genes after an acute load of virgin olive oil. OMICS 2009; 13:431-438.

28. Konstantinidou V, Covas MI, Muñoz-Aguayo D, et al. In vivo nutrigenomic effects of virgin olive oil polyphenols within the frame of the Mediterranean diet: a randomized controlled trial. FASEB J 2010; 24:2546-2557.

29. Ross R. Atherosclerosis--an inflammatory disease. N Engl J Med 1999; 340:115126. 
30. Soran H, Schofield JD, Liu Y, Durrington PN. How HDL protects LDL against atherogenic modification: paraoxonase 1 and other dramatis personae. Curr Opin Lipidol 2015; 26:247-256.

31. Arbonés-Mainar JM, Navarro MA, Carnicer R, et al. Accelerated atherosclerosis in apolipoprotein E-deficient mice fed Western diets containing palm oil compared with extra virgin olive oils: A role for small, dense high-density lipoproteins Atherosclerosis 2007; 194:372-382.

32. Rosenblat M, Volkova N, Coleman R, et al. Antiatherogenicity of extra virgin olive oil and its enrichment with green tea polyphenols in the atherosclerotic apolipoprotein-E-deficient mice: enhanced macrophage cholesterol efflux. $J$ Nutr Biochem 2008; 19:514-523.

33. Bayram B, Ozcelik B, Grimm S, et al. A diet rich in olive oil phenolics reduces oxidative stress in the heart of SAMP8 mice by induction of Nrf2-dependent gene expression. Rejuvenation Res 2012; 15:71-81.

34. Valls RM, Farràs M, Suárez M, et al. Effects of functional olive oil enriched with its own phenolic compounds on endothelial function in hypertensive patients. A randomised controlled trial. Food Chem 2015; 167:30-35.

35. Moreno-Luna R, Muñoz-Hernandez R, Miranda ML, et al. Olive Oil Polyphenols Decrease Blood Pressure and Improve Endothelial Function in Young Women with Mild Hypertension Am J Hypertens 2012; 25:1299-1304.

36. Schwingshackl L, Hoffmann G. Mediterranean dietary pattern, inflammation and endothelial function: a systematic review and meta-analysis of intervention trials. Nutr Metab Cardiovasc Dis 2014, 24:929-939.

37. Meza-Miranda ER, Rangel-Zúñiga OA, Marín C, et al. Virgin olive oil rich in phenolic compounds modulates the expression of atherosclerosis-related genes in vascular endothelium. Eur J Nutr 2015 [In press].

38. Richard C, Couture P, Desroches S, et al. Effect of an isoenergetic traditional Mediterranean diet on the high-density lipoprotein proteome in men with the metabolic syndrome. J Nutrigenet Nutrigenomics 2014; 7:48-60.

39. Otvos JD, Collins D, Freedman DS, et al. Low-density lipoprotein and highdensity lipoprotein particle subclasses predict coronary events and are favorably changed by gemfibrozil therapy in the Veterans Affairs High-Density Lipoprotein Intervention Trial. Circulation 2006; 113:1556-1563. 
40. Damasceno NRT, Sala-Vila A, Cofán M, et al. Mediterranean diet supplemented with nuts reduces waist circumference and shifts lipoprotein subfractions to a less atherogenic pattern in subjects at high cardiovascular risk. Atherosclerosis 2013; 230:347-353.

41. López-Miranda J, Gómez P, Castro P, et al. Mediterranean diet improves low density lipoprotein susceptibility to oxidative modifications. Med Clin (Barc) $2000 ; 115: 361-365$.

42. Derouiche A, Cherki M, Drissi A, et al. Nutritional Intervention Study with Argan Oil in Man: Effects on Lipids and Apolipoproteins Ann Nutr Metab 2005; 49:196-201.

43. Martínez-Beamonte R, Navarro MA, Acin S, et al. Postprandial changes in high density lipoproteins in rats subjected to gavage administration of virgin olive oil. PLoS One 2013; 8:e55231.

44. Richard C, Couture P, Desroches S, et al. Effect of an isoenergetic traditional Mediterranean diet on apolipoprotein A-I kinetic in men with metabolic syndrome. Nutr J 2013; 12:76.

45. Pirillo A, Norata GD, Catapano AL. High-density lipoprotein subfractions--what the clinicians need to know. Cardiology 2013; 124:116-125.

46. Jones JL, Comperatore M, Barona J, et al. A Mediterranean-style, low-glycemicload diet decreases atherogenic lipoproteins and reduces lipoprotein (a) and oxidized low-density lipoprotein in women with metabolic syndrome. Metabolism 2012; 61:366-372.

47. Mangas-Cruz MA, Fernández-Moyano A, Albi T, et al. Effects of minor constituents (non-glyceride compounds) of virgin olive oil on plasma lipid concentrations in male Wistar rats. Clin Nutr 2001; 20:211-215.

48. De la Llera-Moya M, Drazul-Schrader D, Asztalos BF, et al. The ability to promote efflux via ABCA1 determines the capacity of serum specimens with similar high-density lipoprotein cholesterol to remove cholesterol from macrophages. Arterioscler Thromb Vasc Biol 2010; 30:796-801.

49. Sankaranarayanan S, Oram JF, Asztalos BF, et al. Effects of acceptor composition and mechanism of ABCG1-mediated cellular free cholesterol efflux. J Lipid Res 2009; 50:275-284. 
50. Alwaili K, Bailey D, Awan Z, et al. The HDL proteome in acute coronary syndromes shifts to an inflammatory profile. Biochim Biophys Acta 2012;

455 1821:405-415. 
FIGURE TITLES

457

$458 \quad$ Figure 1.

459

460 Heading: Olive oil phenolic compounds and HDL function: hypothetical mechanisms.

461 Legend: The consumption of olive oil phenolic compounds enhances HDL cholesterol

462 efflux capacity, HDL antioxidant capacities and some HDL anti-inflammatory

463 properties. Olive oil phenolic compounds increase cholesterol efflux capacity by

464 improving HDL size distribution, increasing the gene expression of cholesterol

465 transporters, and enhancing ApoA-I function and HDL fluidity (in both cases due to a

466 better HDL oxidative/inflammatory status and changes in HDL composition). HDL

467 antioxidant capacity is incremented after the consumption of virgin olive oil through the

468 preservation of ApoA-I and PON1 function, and an increase in LCAT levels. Finally,

469 HDL anti-inflammatory properties are also augmented, mainly because of the increase

470 in HDL antioxidant function.

471 Continuous lines reflect well-stablished relations between variables, and discontinuous

472 lines hypothetical associations. 
